\title{
"Decoupling" constraints on massless composite particles
}

\author{
John Preskill \\ Lyman Laboratory of Physics, Harvard University, Cambridge, Massachusetts 02138 \\ Steven Weinberg* \\ Department of Physics, University of Texas, Austin, Texas 78712
}

(Received 5 January 1981)

\begin{abstract}
It is pointed out that the use of the "decoupling" constraints on the spectrum of composite massless particles is not justified without further assumptions. There is an alternative condition, whose use would not be subject to the same criticisms, which would lead to the same constraints as the decoupling condition, and which would lead to other results as well, for instance that the nonchiral global symmetries in quantum chromodynamics (QCD) with $n$ massless flavors can not be spontaneously broken. However, this condition is found to be violated in a specific model. It is still an open possibility that the chiral symmetries of QCD are unbroken for $n$ not a multiple of 3 .
\end{abstract}

Composite spin- $\frac{1}{2}$ fermions of zero mass can occur naturally in gauge theories with unbroken global chiral symmetries. Recently, 't Hooft has proposed conditions ${ }^{1}$ on the spectrum of massless bound states in these theories that would require that massless composite particles actually do occur as long as the global chiral symmetries are unbroken. In many cases these conditions are so stringent that they cannot be satisfied at all, which would imply that these symmetries are spontaneously broken.

The first of 't Hooft's conditions is that the massless composite particles must reproduce the same Adler-Bell-Jackiw ${ }^{2}$ anomalies as are produced by the elementary fermions in the currents of all unbroken global chiral symmetries. This condition can probably be proved as a necessary consequence of the symmetry, analyticity, and high-energy behavior of gauge theories, ${ }^{3}$ and it tells us that massless composite particles must occur if the chiral symmetries are unbroken, but by itself it does not in most cases place severe restrictions on the spectrum of massless composite particles that can occur.

This Communication concerns the second of 't Hooft's conditions, known as the "decoupling condition." This condition was introduced by 't Hooft as an example of a subsidiary condition that can be used to pick out a particular set of massless composite particles from among all those sets which satisfy the anomaly condition. It is the combination of the decoupling condition with the anomaly condition that was found in Ref. 1 to be so restrictive that it could be concluded in many cases that chiral symmetries must be spontaneously broken. In brief, the conclusion reached here is that the use of constraints derived from the decoupling condition is not justified; that this condition may be replaced with a stronger condition, the "persistent-mass condition," whose use would not be subject to the same criti- cisms and which would lead to the same conclusions; but that at least in some models the persistent-mass condition is not valid either.

In its original formulation, the decoupling condition requires that the composite particles of any gauge theory must be such that when one of the elementary fermions in the theory is given a large intrinsic mass, the remaining unbroken chiral symmetries permit all composite particles containing this fermion also to get a large mass. For instance, in a gauge theory in which both left- and right-handed fermions form $n$ equivalent complex representations of the gauge group ("flavors"), the Lagrangian in the absence of bare masses or scalar fields will automatically have a global $\mathrm{SU}(n)_{L} \times \mathrm{SU}(n)_{R} \times \mathrm{U}(1)_{V}$ symmetry. If this symmetry is unbroken, there will be a set of massless spin- $\frac{1}{2}$ composite fermions ${ }^{4}$ forming representations $\left(D_{L}, D_{R}\right)$ of $\operatorname{SU}(n)_{L}$ $\times \operatorname{SU}(n)_{R} \times \mathrm{U}(1)_{V}$ with $D_{L} \neq D_{R}$. When one of the $n$ fermion flavors is given a large intrinsic mass, the symmetry of the Lagrangian is reduced to $\operatorname{SU}(n-1)_{L}$ $\times \mathrm{SU}(n-1)_{R} \times \mathrm{U}(1) v^{2}$. The decoupling condition requires that the composite particles which contain the heavy quark must not be prevented by this remaining symmetry from becoming heavy.

Stated in this way, the decoupling condition appears as a very plausible consequence of the general idea that particles with large masses should drop out of the theory of lower energy states. ${ }^{5}$ The trouble is that in order to apply this condition as it is applied in Ref. 1, it is necessary to make another assumption, that the pattern of spontaneous symmetry breaking and the representation content of bound massless particles do not change when one of the elementary fermions is given a very large intrinsic mass. For instance, in applying the decoupling condition to the $n$ flavor gauge theory mentioned above, it was tacitly assumed in Ref. 1 not only that the $\operatorname{SU}(n)_{L} \times \operatorname{SU}(n)_{R}$ 
$\times \mathrm{U}(1)_{v}$ global symmetry of the Lagrangian is not spontaneously broken when all fermion masses vanish, but also that the $\mathrm{SU}(n-1)_{L} \times \mathrm{SU}(n-1)_{R}$ $\times U(1) V^{2}$ symmetry of the Lagrangian with one massive quark remains not spontaneously broken when the massive quark becomes very heavy. Otherwise, the $\mathrm{SU}(n-1)_{L} \times \mathrm{SU}(n-1)_{R} \times \mathrm{U}(1)_{V}{ }^{2}$-representation content of the composite particles would, of course, be irrelevant in determining whether composite particles containing the heavy quark become heavy.

It seems to us quite possible that the underlying idea of decoupling is correct, but that its application in Ref. 1 is invalid, because a phase transition may occur when one of the elementary fermion masses becomes sufficiently large. This phase transition could involve either a change in the pattern of spontaneous symmetry breaking or a change in the set of massless composite fermions unaccompanied by a change in symmetry, or both. For instance, in the $n$-flavor gauge theory discussed above, if the $\mathrm{SU}(n)_{L} \times \mathrm{SU}(n)_{R} \times \mathrm{U}(1)_{V}$ symmetry of the Lagrangian is not spontaneously broken when all of the fermions are massless, then clearly the remaining $\mathrm{SU}(n-1)_{L} \times \mathrm{SU}(n-1)_{R} \times \mathrm{U}(1)_{V}^{2}$ symmetry is unbroken when one of the fermions is given a very small mass. There may be composite states that contain this massive quark, but are kept massless by this chiral symmetry. For example, in the $n=4$ case there is a simple set of massless composite threefermion states that satisfy the anomaly condition, forming $\mathrm{SU}(4)_{L} \times \mathrm{SU}(4)_{R}$ representations $(4,6)$ $+3(\overline{4}, 1)$ for helicity $+\frac{1}{2}$ and $(6,4)+3(1, \overline{4})$ for helicity $-\frac{1}{2}$. When one of the four quark flavors is given a small mass, the states containing one massive quark form $\mathrm{SU}(3)_{L} \times \mathrm{SU}(3)_{R}$ representations $(3,3)+(1, \overline{3})+3(\overline{3}, 1)$ for helicity $+\frac{1}{2}$ and $(3,3)+(\overline{3}, 1)+3(1, \overline{3})$ for helicity $-\frac{1}{2}$, while the states containing two massive quarks form an $\mathrm{SU}(3)_{L} \times \mathrm{SU}(3)_{R}$ representation $(1,3)$ for helicity $+\frac{1}{2}$ and $(3,1)$ for helicity $-\frac{1}{2}$. Thus as long as $\mathrm{SU}(3)_{L} \times \mathrm{SU}(3)_{R} \times \mathrm{U}(1)_{V}^{2}$ remains unbroken, there will be two massless $(\overline{3}, 1)$ states of helicity $+\frac{1}{2}$ containing one massive quark, and one massless $(1,3)$ state of helicity $+\frac{1}{2}$ containing two massive quarks, plus their parity conjugates. In such cases, the requirement of decoupling must be met by the intervention of a phase transition, at which the $\mathrm{SU}(n-1)_{L} \times \mathrm{SU}(n-1)_{R}$ symmetry becomes broken or the pattern of bound states changes when one of the quark masses becomes sufficiently large.

This discussion suggests an alternative to the decoupling condition, which we will call the "persistent-mass condition." This condition can be stated as follows:

The composite particles of any gauge theory must be such that when one of the elementary fermions in the theory is given any mass, the remaining unbro- ken chiral symmetries permit all composite particles containing this quark also to get some mass.

This condition is clearly stronger than the decoupling condition of Ref. 1 , and is based on intuitive ideas about the masses of composite systems rather than on known properties of quantum field theories. However, the persistent-mass condition can be applied without any assumptions about the stability of the pattern of spontaneous symmetry breaking or the spectrum of bound states as masses tend to infinity. In applying this condition, we need only consider the case when one elementary fermion is given an infinitesimal mass, where we do know the unbroken symmetries of the theory. For instance, in the $n$-flavor gauge theory, if $\mathrm{SU}(n)_{L} \times \mathrm{SU}(n)_{R} \times \mathrm{U}(1)_{V}$ is not spontaneously broken when all fermions are massless, then $\mathrm{SU}(n-1)_{L} \times \mathrm{SU}(n-1)_{R} \times \mathrm{U}(1)_{v}{ }^{2}$ is unbroken when one fermion is given an infinitesimal mass. All the consequences of the decoupling condition derived in Ref. 1 follow also from the persistent-mass condition, because the limit of very large fermion mass was used in Ref. 1 only in justifying the decoupling condition, not in applying it.

The persistent-mass condition leads to other consequences as well, that may be used to judge its validity. In the $n$-flavor gauge theory with zero fermion masses discussed above, the complete spontaneous breakdown of the $\operatorname{SU}(n)_{L} \times \operatorname{SU}(n)_{R}$ symmetry of the Lagrangian would entail the existence of $n^{2}-1$ scalar as well as $n^{2}-1$ pseudoscalar massless Goldstone bosons, all of which are interpreted as composites of quarks and antiquarks. If the $n$ fermion flavors are given small equal intrinsic masses, the $n^{2}-1$ oddparity symmetries of $\mathrm{SU}(n)_{L} \times \mathrm{SU}(n)_{R}$ become intrinsically broken and the corresponding pseudoscalar Goldstone bosons become massive, in accord with the persistent-mass condition. However, the positive-parity $\mathrm{SU}(n)$ generators are not intrinsically broken by equal fermion masses, so the $n^{2}-1$ scalar Goldstone bosons remain massless, in contradiction with the persistent-mass condition. Thus the persistent-mass condition would have as a consequence that although the negative-parity generators of $\mathrm{SU}(n)_{L} \times \mathrm{SU}(n)_{R}$ can be spontaneously broken, the positive-parity $\mathrm{SU}(n)$ subgroup cannot be. In general, the persistent-mass condition implies that any continuous flavor symmetry which allows all flavors to acquire mass cannot be spontaneously broken.

This is a powerful result, and would allow us to explain in quantum chromodynamics (QCD) with three light quarks why the SU(3) of the "eight-fold way" is not spontaneously broken. But before we accept this explanation, let us consider whether these consequences of the persistent-mass condition are actually satisfied in a specific model.

The model considered here is a variant of the old $\mathrm{Nambu}$-Jona-Lasinio (NJL) model $^{6}$ of spontaneous symmetry breaking, but the spontaneously broken 
symmetry is a nonchiral isospin symmetry rather than a chiral symmetry. We consider an isotopic doublet $\psi$ of spin- $\frac{1}{2}$ fields, described by the isospin-conserving Lagrangian

$$
L=-\bar{\psi}\left(\gamma^{\mu} \partial_{\mu}+m_{0}\right) \psi+\frac{1}{2} G(\bar{\psi} \vec{\tau} \psi)^{2}
$$

Here $\vec{\tau}$ are the Pauli isospin matrices, $m_{0}$ is a bare mass matrix that commutes with $\vec{\tau}$, and $G$ is a free coupling constant. As in the NJL model, this Lagrangian is nonrenormalizable, and an ultraviolet cutoff $\Lambda$ is introduced to deal with infinities. Also as in the NJL model, the fermion self-energy is calculated from the one-loop diagram, using the dressed mass matrix $m$ in the virtual fermion propagator. This yields an implicit equation for $m$ :

$$
\begin{array}{r}
m=m_{0}-\frac{i G}{(2 \pi)^{4}} \int d^{4} k[\vec{\tau} \cdot \operatorname{Tr}\{S(k, m) \vec{\tau}\} \\
-\vec{\tau} \cdot S(k, m) \vec{\tau}],
\end{array}
$$

where $S(k, m) \equiv\left(i \gamma_{\mu} k^{\mu}+m\right)^{-1}$ is the fermion propagator. Also, in the first-order approximation for the kernel, the Bethe-Salpeter equation for composite fermionantifermion systems of total four-momentum $p^{\mu}$ is

$$
\begin{array}{r}
\Psi(q ; p)=\frac{i G}{(2 \pi)^{4}} \int d^{4} k[\vec{\tau} \cdot \operatorname{Tr}\{S(k-p) \Psi(k ; p) S(k) \vec{\tau}\} \\
-\vec{\tau} \cdot S(k-p) \Psi(k ; p) S(k) \vec{\tau}]
\end{array}
$$

It is elementary to check in this model that the spontaneous breakdown of isospin conservation implies the existence of massless Goldstone bosons; that is, if $m$ is a solution of Eq. (2) with $[\vec{\tau}, m] \neq 0$, then Eq.

(3) is satisfied for $p^{\mu}=0$, with

$$
\Psi_{a}(q ; 0)=\left[\tau_{a}, m\right] \text {. }
$$

So it is only necessary to find broken-symmetry solutions of Eq. (2) in order to conclude that this model involves massless composite bosons.

Without loss of generality, we can always choose the isospin basis so that

$$
m=m_{S}+m_{V} \tau_{3} \text {. }
$$

Equation (2) then yields two equations

$$
m_{S}=m_{0}-\frac{3 G m_{S}}{8 \pi^{2}} \int_{0}^{\Lambda} \frac{\left(k^{2}+m_{S}^{2}-m_{V}^{2}\right) k^{3} d k}{\left(k^{2}+m_{S}^{2}+m_{V}^{2}\right)^{2}-4 m_{S}^{2} m_{V}^{2}}
$$$$
m_{V}=\frac{9 G}{8 \pi^{2}} m_{V} \int_{0}^{\Lambda} \frac{\left(k^{2}+m_{V}^{2}-m_{S}^{2}\right) k^{3} d k}{\left(k^{2}+m_{S}^{2}+m_{V}^{2}\right)^{2}-4 m_{S}^{2} m_{V}^{2}} .
$$

First let us consider the case $m_{0}=0$. Equation (6) then has the solution $m_{S}=0$, and for $m_{V} \neq 0$, Eq. (7) reduces to

$$
\frac{8 \pi^{2}}{9 G}=\int_{0}^{\Lambda} \frac{k^{3} d k}{\left(k^{2}+m_{V}^{2}\right)}
$$

The right-hand side takes all values from 0 to $\Lambda^{2} / 2$, so Eq. (8) has a solution with $m_{V} \neq 0$ for $G>16 \pi^{2} / 9 \Lambda^{2}$. Thus for $m_{0}=0$, isospin is broken for sufficiently strong coupling, and the massless fermions form massless bound states.
Now let us turn on the intrinsic fermion masses. Since we found a solution with $m_{V} \neq 0$ for $m_{0}=0$, we would expect such a solution to exist for at least a small range of values of $m_{0}$, and this is borne out by detailed calculation: The symmetry-breaking mass term $m_{V}$ is shifted by an amount of order $m_{0}^{2}$, and thus remains nonzero for $m_{0}$ sufficiently small. It follows then that the Bethe-Salpeter equation (3) has the zero-mass solution (4) for such values of $m_{0}$. The "persistent mass" condition is definitely violated here; massless composite particles are formed from constituents with nonzero intrinsic masses. This counter-intuitive result is possible here because, when we vary the intrinsic fermion mass $m_{0}, m_{S}$ and $m_{V}$ are forced by Eqs. (6) and (7) to adjust in such a way as to keep the composite boson massless.

What about decoupling? For any fixed value of $G \Lambda^{2}$ greater than $16 \pi^{2} / 9$, there is a critical value of $\left|m_{0}\right|$, at which $m_{V}$ drops to zero. (For instance, for $G \Lambda^{2}>16 \pi^{2} / 9$, the critical value of $\left|m_{0}\right|$ is 0.48 $G \Lambda^{3} / 8 \pi^{2}$.) At larger values of $\left|m_{0}\right|$, isospin symmetry is restored, and there no longer is a bound state at zero mass. The phase transition is second order, and the spectrum of the model varies continuously, but nonanalytically, through the transition. Eventually, for $\left|m_{0}\right|$ sufficiently large, the mass of any fermion-antifermion state simply approaches $2\left|m_{0}\right|$. This is in accord with our general expectations about the decoupling of heavy particles, but these expectations cannot be relied on in this model to tell us anything about the spectrum of composite particles when $m_{0}=0$, because a phase transition separates the regions of large and small $m_{0}$.

Of course, this model has many special features, and it is possible that the persistent-mass condition might be valid in more realistic theories, like QCD. The example given here only shows that we cannot without further argument rely on the intuitive idea that massive constituents make massive composites.

If we do give up the constraints that have been derived from the persistent-mass or decoupling conditions, we find a wide variety of possible sets of massless composite fermions in theories with unbroken chiral symmetries. For instance, in QCD with $n$ quark flavors, the anomaly condition can be satisfied by massless composite fermions of helicity $+\frac{1}{2}$ belonging to the $\mathrm{SU}(n)_{L} \times \mathrm{SU}(n)_{R}$ representations:

$$
\begin{array}{ll}
n=2: & (2,1), \\
n=4: & (4,6)+3(\overline{4}, 1), \\
n=5: & (5,10)+2(\overline{10}, 1), \\
n=7: & 3(84,1)+2(35,1)+4(7,28)+(7,21), \\
n=8: & (120,1)+6(1,56)+(8,36)+4(28,8), \\
n=10: & 3(220,1)+2(120,1)+2(10,55) \\
& +(45,10)+2(1,330) \\
n=11: & 5(165,1)+(11,66)+5(11,55)+(440,1), \\
n=13: & (455,1)+5(1,286)+(13,91)+4(78,13), \\
n=14: & 3(1,560)+2(1,364)+3(14,91)+4(910,1), \\
n=16: & 4(816,1)+3(560,1)+(16,136) \\
& +2(120,16)+4(1,1360),
\end{array}
$$


plus their parity conjugates. There are no sets of composite particles that are consistent with the anomaly condition when $n$ is a multiple of 3 , because the $\mathrm{SU}(n)_{L} \times \mathrm{SU}(n)_{L} \times \mathrm{U}(1)_{V}$ anomaly in this case is a multiple of 3 for any color-singlet states, while the elementary quarks in QCD give an anomaly of unity. But there are an infinite number of possible sets of massless composite fermions that do satisfy the anomaly conditions, for all values of $n$ that are not multiples of 3 .

From 't Hooft's observation ${ }^{1}$ that there are no sets of massless composite fermions that satisfy all the constraints derived from the decoupling and anomaly conditions in QCD with $n>2$, we learn that completely unbroken chiral symmetry in QCD would imply the existence of a phase transition. For suppose that the chiral symmetries of QCD with some number $n>2$ of massless flavors are not spontaneously broken. Then the constraints derived in Ref. 1 from the decoupling condition must be violated, and we have seen that this can happen only if a phase transition occurs when one of the $n$ quarks is given a sufficiently large intrinsic mass while the other $n-1$ quarks remain massless.

It is instructive to speculate about the nature of this phase transition. One might infer from its existence that the $\mathrm{SU}(n-1)_{L} \times \mathrm{SU}(n-1)_{R}$ symmetry of the theory with one massive quark becomes spontaneously broken when the mass of this quark is sufficiently large. But when one quark's mass becomes very large, all others remaining zero, QCD with $n$ massless flavors goes over to QCD with $n-1$ massless flavors. Thus we would conclude that, if the chiral symmetries of QCD are not spontaneously broken for $n$ massless flavors, then they must be spontaneously broken for $n-1$ massless flavors. Furthermore, Bars ${ }^{7}$ has generalized the analysis of Ref. 1 to the case in which $k$ flavors simultaneously receive the intrinsic mass $m$, while $n-k$ flavors remain massless. Again one finds that, if the chiral symmetries are not spontaneously broken in $n$-flavor QCD, then a phase transition must occur at a critical value of $m$, from which one might infer that the chiral symmetries of QCD must be spontaneously broken for $n-k$ flavors. As Bars ${ }^{7}$ points out, this line of reasoning leads to the conclusion that the chiral symmetries can escape spontaneous breakdown for at most one value of $n$.

However, the existence of a phase boundary separating the theories with large and small quark mass $m$ does not necessarily imply that the $\mathrm{SU}(n-k)_{L}$ $\times \mathrm{SU}(n-k)_{R} \times \mathrm{U}(1)_{V}$ chiral symmetry cannot be unbroken for both large and small $\mathrm{m}$. The phase transition could be a discontinuous change in the spectrum of the theory not associated with a change in symmetry, an accidental degeneracy of two chiralinvariant vacuums at some value of $m$. Alternatively, there may be two (or more) critical values of $m$ at which chiral symmetry is first spontaneously broken and then restored as $m$ increases, in which case the transitions may be either first order or second order. Hence, it is still an open possibility that the chiral symmetries of QCD are not spontaneously broken for more than one value of $n$, including perhaps consecutive values of $n$ which are not multiples of 3 .

The chiral symmetries of QCD can escape spontaneous breakdown for some $n$ only if the qualitative behavior of QCD is sensitively dependent on $n$. Chiral symmetries are spontaneously broken if $n$ is a multiple of $3,{ }^{8}$ or if the number of colors is even or large. ${ }^{9}$ The persistent-mass condition, if valid, would allow us to conclude that the chiral symmetries of QCD for any $n>2$ are spontaneously broken and that the nonchiral symmetries are not spontaneously broken. However, we believe that the persistentmass condition has no general quantum fieldtheoretic basis. Instead, a different criterion is needed to determine how $n$-flavor QCD actually chooses to realize its global flavor symmetries.

We are grateful to Itzhak Bars, Sidney Coleman, Estia Eichten, Howard Georgi, Bob Holdom, Roman Jackiw, Leonard Susskind, and Edward Witten for valuable discussions. Research of one of us (J.P.) was supported in part by the Harvard Society of Fellows.
"On leave 1980-81 from the Department of Physics, Harvard University, Cambridge, Massachusetts 02138.

${ }^{1} \mathrm{G}$. ' $\mathrm{t}$ Hooft, lecture given at the Cargèse Summer Institute, 1979 (unpublished). Also see S. Dimopoulos, S. Raby, and L. Susskind, Nucl. Phys. B173, 208 (1980); S. Coleman and E. Witten, Phys. Rev. Lett. 45, 100 (1980); Y. Frishman, A. Schwimmer, T. Banks, and S. Yankielowicz, Nucl. Phys. B177, 157 (1981); A. Zee, Univ. of Pennsylvania report, 1980 (unpublished); R. Barbieri, L. Maiani, and R. Petronzio, Phys. Lett. 96B, 63 (1980); G. Farrar, ibid. 96B, 273 (1980); R. Chanda and P. Roy, ibid. 99B, 453 (1981).

${ }^{2}$ S. L. Adler, Phys. Rev. 177, 2426 (1969); J. S. Bell and R. Jackiw, Nuovo Cimento 60A, 47 (1969).

${ }^{3} \mathrm{~S}$. Coleman and B. Grossman (private communication); Frishman et al., Ref. 1.

${ }^{4}$ The proof that these fermions must have spin $\frac{1}{2}$ is given by
S. Weinberg and E. Witten, Phys. Lett. 96B, 59 (1980).

Also see L. Durand, Phys. Rev. 128, 434 (1962).

${ }^{5}$ T. Aprelquist and J. Carazzone, Phys. Rev. D 11, 2856 (1975).

${ }^{6}$ Y. Nambu and G. Jona-Lasinio, Phys. Rev. 122, 345 (1961).

${ }^{7}$ I. Bars, Yale Report No. YTP 81-09, 1981 (unpublished).

${ }^{8} \mathrm{We}$ cannot rule out the possibility that partial breaking to a chiral subgroup of the full chiral group occurs in this case, or for the other values of $n$. Another interesting possibility is that the unbroken chiral symmetry is a discrete symmetry which still suffices to keep some composite fermions massless.

${ }^{9} \mathrm{~S}$. Coleman and E. Witten, Ref. 1. It should be noted, though, that if massless baryons occur for an arbitrarily large number of colors $N_{c}$, then the large- $N_{c}$ limit is not expected to exist, because massless baryon pairs couple to flavor currents with a strength of order $N_{c}$. See E. Witten, Nucl. Phys. B160, 57 (1979). 